\title{
The log-conformation tensor approach in the finite-volume method framework
}

\author{
A. Afonso ${ }^{a}$, P.J. Oliveira ${ }^{b}$, F.T. Pinho ${ }^{c, d}$, M.A. Alves ${ }^{a, *}$ \\ a Departamento de Engenharia Química, Centro de Estudos de Fenómenos de Transporte, Faculdade de Engenharia da Universidade do Porto, \\ Rua Dr. Roberto Frias, 4200-465 Porto, Portugal \\ ${ }^{\mathrm{b}}$ Departamento de Engenharia Electromecânica, Unidade de Materiais Têxteis e Papeleiros, Universidade da Beira Interior, 6201-001 Covilhã, Portugal \\ c Universidade do Minho, Largo do Paço, 4704-553 Braga, Portugal \\ d Centro de Estudos de Fenómenos de Transporte, Faculdade de Engenharia da Universidade do Porto, Rua Dr. Roberto Frias, $4200-465$ Porto, Portugal
}

\section{A R T I C L E I N F O}

\section{Article history:}

Received 5 May 2008

Received in revised form

19 September 2008

Accepted 22 September 2008

\section{Keywords:}

Finite-volume method

Log-conformation tensor

Viscoelastic fluid

Cylinder flow

\begin{abstract}
A B S T R A C T
The log-conformation formulation, proposed by Fattal and Kupferman [J. Non-Newt. Fluid Mech. 123 (2004) 281], has helped to provide further insights into the High-Weissenberg Number Problem. In this work, we investigate the performance of the log-conformation formulation in the Finite Volume Method (FVM) framework for creeping flows of viscoelastic fluids in steady and unsteady flows around a confined cylinder. The Oldroyd-B and Phan-Thien-Tanner (PTT) constitutive equations were used to assess the effect of different rheological behaviour on the flow patterns and solution stability. The calculation of the polymer stress contribution is carried out with both the standard technique and with the logconformation methodology. For all test cases, up to the critical conditions when both methods converge to a steady solution, the use of the log-conformation technique provides solutions with similar accuracy as the standard approach. In terms of stability the log-conformation formulation is found to be significantly more robust, and solutions could be obtained at higher Deborah number flows.
\end{abstract}

(c) 2008 Elsevier B.V. All rights reserved.

\section{Introduction}

A Finite Volume Method (FVM) is applied to the numerical simulation of laminar viscoelastic flow around a confined cylinder using the Oldroyd-B model [1] and the Phan-Thien-Tanner (PTT) model $[2,3]$ with linear stress coefficient kernel. The Oldroyd-B fluid was selected for this study, in order to allow direct comparison with previous works (e.g. Alves et al. [4]), and also to analyze the applicability and benefits of using the matrix logarithm transformation [5] for Maxwell-type models in a smooth flow (i.e. without geometric singularities).

The flow around a confined cylinder is a usual benchmark testcase in computational rheology. It is representative of fundamental flow dynamics of viscoelastic fluids around solid bodies and it can be encountered in many engineering processes. This flow has been studied experimentally by McKinley et al. [6], Baaijens et al. [7] and Shiang et al. [8,9]. Owens and Phillips [10] documented and summarized the main numerical results, focusing primarily on the Oldroyd-B model. Although the flow around a confined cylinder is classified as a smooth flow, its numerical calculation presents some difficulties associated with the development of thin stress layers

\footnotetext{
* Corresponding author.

E-mail addresses: aafonso@fe.up.pt (A. Afonso), pjpo@ubi.pt (P.J. Oliveira), fpinho@fe.up.pt (F.T. Pinho), mmalves@fe.up.pt (M.A. Alves).
}

on the cylinder sidewall and along the centreline in the cylinder rear wake, imposing a limiting value to the Deborah number for which steady solutions can be obtained. In fact, the simulations for the cylinder flow with the Upper-Convected Maxwell (UCM) or Oldroyd-B fluids are somehow limited by the so-called HighWeissenberg Number Problem (HWNP; like the Deborah number, the Weissenberg number measures the flow elasticity). A breakdown in the calculations is found at a limiting Deborah number below 1 for the UCM fluid whereas for the Oldroyd-B fluid the breakdown occurs at higher Deborah numbers depending on the solvent viscosity ratio.

In order to get further insights into the HWNP, this work implements the methodology recently proposed by Fattal and Kupferman [5], the so-called matrix-logarithm or log-conformation formulation (nomenclature used heretoforth) of the viscoelastic constitutive equations. This is based on a reformulation of the constitutive law in terms of the matrix logarithm of the conformation tensor. According to Fattal and Kupferman [5], taking the logarithm of the conformation tensor reduces its very large (often exponential) variation (e.g. in stagnation points, or near the walls). The new variable can be more accurately approximated by a polynomial interpolation, and the method also preserves the positive definiteness of the conformation tensor [5,11-18]. A similar logarithm transformation was used previously for scalar quantities in mass transfer problems by Miranda and Campos [19], who applied a simple logarithmic variable transformation of the solute concentra- 
tion in the solution of laminar flow and solute transport equations in a parallel plate device with permeable walls. This variable transformation improved their finite difference method allowing the use of a larger grid spacing without loss of accuracy. A logarithm transformation has also been used in turbulence modelling using the $k-\varepsilon$ formalism [20]. The model is rewritten in terms of the variables $\log (k)$ and $\log (\varepsilon)$, and when $k$ and $\varepsilon$ variables are recovered the result is always positive.

Fattal and Kupferman [5] reported a breakthrough in the HWNP in their numerical simulations with the Finitely Extensible Nonlinear Elastic model using the Chilcott-Rallison approximation (FENE-CR) in a two-dimensional lid-driven cavity flow. Later, Fattal and Kupferman [11] applied the log-conformation scheme to the flow of an Oldroyd-B fluid in the same geometry, using a multigrid solver, and reported the ability to perform stable simulations at large values of the Weissenberg number. They proposed a stability criterion and stated that this condition may be very restrictive when convection is weak and in the presence of large deformation rates, as in the flow around sharp corners. Recently, Pan and Hao [12] performed numerical simulations of the lid-driven cavity creeping flow for an Oldroyd-B fluid, using the finite element method (FEM). They also relied on the log-conformation technique, and found that this methodology is stable at higher Weissenberg numbers, and presented solutions up to $D e=3$.

Hulsen et al. [13] were the first to implement the logconformation methodology within the FEM framework and applied it to the benchmark flow of Oldroyd-B and Giesekus fluids past a confined cylinder. They reported an almost unbounded convergence limit for the Giesekus model, whereas for the Oldroyd-B fluid the solution became unsteady at higher Deborah numbers, while exhibiting symptoms of strong mesh dependency particularly in the stress fields near the cylinder. Kwon [14] investigated numerically the planar 4:1 contraction flow using the Leonov constitutive equation and found stabler computations when using the log-conformation method than with the conventional approach. Kwon [14] also concluded that this new method may only work efficiently for constitutive equations that are proven globally stable and that the stability constraint has to be taken into serious consideration. In a sequel, Yoon and Kwon [15] obtained solutions for Deborah numbers in excess of 100 using finer meshes. These authors also presented solutions for the 4:1:4 contraction/expansion flow and obtained converged solutions for Deborah numbers above 10 . However, in both geometries the convergence limits decreased with mesh refinement. More recently, Kwon [16] extended the log-conformation formulation to the calculations of flows of viscoelastic fluids in a channel obstructed by an asymmetric array of cylindrical obstacles. The Leonov model was employed, and stable solutions were obtained with an apparent unbounded convergence limit when the retardation parameter that specifies the solvent viscosity contribution was 0.5 . In contrast, in the absence of a solvent contribution to the Leonov model, the limiting Deborah number becomes finite, between 4.5 and 20.

Coronado et al. [17] used an alternate implementation of the log-conformation formulation in their simulations of the planar Couette flow and flow past a cylinder in a channel, for several viscoelastic fluids. These were modelled by a generalized constitutive equation formulated in terms of the conformation tensor. Their results demonstrated that this methodology works well and improves the numerical stability at higher De, especially in the flow past a confined cylinder, where the maximum De limit was extended to 1.0 as compared to 0.7 obtained with the standard DEVSS-TG/SUPG method. The results were also in good agreement with those presented by Hulsen et al. [13]. Recently, Guénette et al. [18] performed simulations of the flow of a viscoelastic fluid around a confined cylinder using the Oldroyd-B and Giesekus models. They used a FEM, with an anisotropic adaptive remeshing method, based on the log-conformation formulation, and showed that convergence with mesh refinement is possible for the Oldroyd-B model up to at least $D e=0.7$, although at $D e=0.8$ mesh convergence was lost.

The remaining of this paper is organized as follows: after presenting the governing equations, the log-conformation modified constitutive equation is described. Then, we explain how the positive definiteness of the conformation tensor is assessed. Prior to the presentation of results the numerical method is briefly described and the geometry and computational meshes used are presented. The paper ends with the main conclusions.

\section{Governing equations}

To simulate the steady incompressible flow of viscoelastic fluids, the following set of governing equations needs to be solved: conservation of mass,

$\nabla \cdot \mathbf{u}=0$,

conservation of momentum,

$\rho \frac{\mathrm{D} \mathbf{u}}{\mathrm{D} t}=-\nabla p+\beta \eta_{o} \nabla^{2} \mathbf{u}+\frac{\eta_{o}}{\lambda}(1-\beta) \nabla \cdot \mathbf{A}$,

containing an additive term which either obeys the Oldroyd-B or the PTT model and a constitutive equation describing the evolution of the conformation tensor, $\mathbf{A}$,

$\lambda \stackrel{\nabla}{\lambda}=-Y(\operatorname{tr} \mathbf{A})(\mathbf{A}-\mathbf{I})$

where $\mathbf{I}$ is the unit tensor, $\mathbf{u}$ is the velocity vector, $p$ is the pressure, $\lambda$ is the relaxation time of the polymer, and $\mathbf{A}$ represents Oldroyd's upper-convected derivative of $\mathbf{A}$, given by

$\stackrel{\nabla}{\mathbf{A}}=\frac{\mathrm{DA}}{\mathrm{D} t}-\mathbf{A} \cdot \nabla \mathbf{u}-\nabla \mathbf{u}^{\mathrm{T}} \cdot \mathbf{A}$

The total fluid extra-stress is the sum of the solvent and polymer stress contributions, and the viscosity ratio, $\beta$, is defined as the ratio between the Newtonian solvent viscosity, $\eta_{s}$, and the total zero shear-rate viscosity, $\eta_{0}$,

$\beta \equiv \frac{\eta_{s}}{\eta_{0}}=\frac{\eta_{s}}{\eta_{s}+\eta_{P}}$

where $\eta_{P}$ is the coefficient of viscosity of the polymer.

In its general form function $Y(\operatorname{tr} \mathbf{A})$ for the PTT model is exponential [3] but in this work we use its linear form, $Y(\operatorname{tr} \mathbf{A})=1+\varepsilon(\operatorname{tr} \mathbf{A}-3)$ [2]. When $Y(\operatorname{tr} \mathbf{A})=1$ (i.e. for $\varepsilon=0$ ) the Oldroyd-B model is recovered. The non-unitary form of $Y(\operatorname{tr} \mathbf{A})$ for the PTT model with a Newtonian solvent imparts shear-thinning behaviour to the shear viscosity of the fluid and bounds its extensional viscosity. The constitutive law written in terms of the conformation tensor $\mathbf{A}$, can be explicitly formulated as a function of the polymer contribution to the extra-stress tensor, $\boldsymbol{\tau}$, with the following relation valid for both models,

$\boldsymbol{\tau}=\frac{\eta_{P}}{\lambda}(\mathbf{A}-\mathbf{I})$.

The use of these governing equations in terms of $\boldsymbol{\tau}$ was followed in previous works [4,21-25], where the numerical methodology was extensively validated in the framework of FVM. This approach is known as the standard method, and is not used here except in some comparisons to assess the performance of the new logconformation method. In such comparison the standard method will be based on the polymer stress given by Eqs. (3) and (5). 


\subsection{The log-conformation representation}

As mentioned above, Fattal and Kupferman [5] suggested a simple tensor-logarithmic transformation of the conformation tensor for differential viscoelastic constitutive equations, which can be applied to a wide variety of constitutive laws. The core feature of the transformation is the decomposition of the velocity gradient, $\nabla \mathbf{u}^{\mathrm{T}}$, into a traceless extensional component, $\mathbf{E}$, and a pure rotational component, $\mathbf{R}$. With this decomposition, the evolution Eq. (3), can be re-written as [5]:

$$
\frac{\partial \mathbf{A}}{\partial t}+(\mathbf{u} \cdot \nabla) \mathbf{A}-(\mathbf{R A}-\mathbf{A R})-2 \mathbf{E A}=\frac{Y(\operatorname{tr} \mathbf{A})}{\lambda}(\mathbf{I}-\mathbf{A})
$$

In the log-conformation representation the evolution Eq. (6) is replaced by an equivalent evolution equation for the logarithm of the conformation tensor, $\boldsymbol{\Theta}=\log \mathbf{A}$, benefiting from the fact that A is a symmetric positive definite (SPD) matrix, and thus can be diagonalized into the form [5]:

$\mathbf{A}=\boldsymbol{\Omega} \mathbf{D} \boldsymbol{\Omega}^{\mathrm{T}}$,

where $\boldsymbol{\Omega}$ is an orthogonal matrix that consist of the eigenvectors of matrix $\mathbf{A}$, and $\mathbf{D}$ is a diagonal matrix made with the corresponding three distinct eigenvalues of matrix $\mathbf{A}$. The transformation from Eq. (6) to an equation for $\Theta=\log \mathbf{A}$ is described in [5], and leads to

$$
\frac{\partial \boldsymbol{\Theta}}{\partial t}+(\mathbf{u} \cdot \nabla) \boldsymbol{\Theta}-(\mathbf{R} \boldsymbol{\Theta}-\boldsymbol{\Theta} \mathbf{R})-2 \mathbf{E}=\frac{Y\left[\operatorname{tr}\left(\mathrm{e}^{\boldsymbol{\Theta}}\right)\right]}{\lambda}\left(\mathrm{e}^{-\boldsymbol{\Theta}}-\mathbf{I}\right)
$$

To recover $\boldsymbol{A}$ from $\boldsymbol{\Theta}$ the inverse transformation $\mathbf{A}=\mathrm{e}^{\boldsymbol{\Theta}}$ is used when necessary.

The positive definiteness of the conformation tensor is crucial for the well posedness of the evolution equation. In this work, the positive definiteness is assessed by checking if the determinant of $\mathbf{A}$ is positive (a necessary condition for $\mathbf{A}$ being positive definite). For the Oldroyd-B model, $\operatorname{det}(\mathbf{A}) \geq 1$ as shown by Hulsen [26].

\section{Numerical method and computational meshes}

In the past, our group adapted a Newtonian FVM to calculate viscoelastic flows where the polymer contribution to the extra-stress tensor was described by one of various differential rheological constitutive equations [4,21-25]. This fully implicit FVM is based on a time-marching pressure-correction algorithm, formulated with the collocated variable arrangement, as explained in detail in Oliveira et al. [21] and Alves et al. [22,23]. Here, the methodology is adapted to the log-conformation procedure and the corresponding modifications are explained below.

The governing equations are first transformed to a nonorthogonal system, but keeping the Cartesian velocity and conformation/log-conformation components. The logconformation tensor evolution Eq. (8) can thus be written into a general non-orthogonal coordinate $\operatorname{system}\left(\varsigma_{1}, \varsigma_{2}, \varsigma_{3}\right)$ as

$$
\begin{aligned}
\frac{\partial J \Theta_{i j}}{\partial t}+\frac{\partial}{\partial \varsigma_{l}}\left(u_{k} \beta_{l k} \Theta_{i j}\right)= & J\left(R_{i k} \Theta_{k j}-\Theta_{i k} R_{k j}\right) \\
& +2 J E_{i j}+Y\left(A_{k k}\right) \frac{J}{\lambda}\left(\mathrm{e}^{-\Theta_{i j}}-\delta_{i j}\right)
\end{aligned}
$$

where $J$ is the Jacobian of the transformation $x_{k}=x_{k}\left(\varsigma_{l}\right)$ and $\beta_{l k}$ are metric coefficients, defined by the cofactor of $\partial x_{k} / \partial \varsigma_{l}$. After integration over the control volumes forming the computational mesh, and in time over a time step $(\delta t)$, the $\beta_{l k}$ metric coefficients are replaced by area components (index $k$ ) of the surface whose normal vector points towards direction $l$, the Jacobian $J$ is replaced by the computational cell volume $V$, and the derivatives $\partial / \partial \varsigma_{l}$ become differences between values along direction $l$. More details can be found in Oliveira et al. [21].
After the discretization, the various terms are assembled, to obtain a system of algebraic equations of the form

$a_{P}^{\Theta} \Theta_{i j, P}-\sum_{F} a_{F}^{\Theta} \Theta_{i j, F}=S_{\Theta_{i j}}+\frac{\lambda_{P} V_{P}}{\delta t} \Theta_{i j, P}^{0}$,

where $\Theta_{i j, P}^{0}$ refers to the $i j$ component of the log-conformation tensor at the previous time level, $a_{P}^{\Theta}$ represents the central coefficient, $a_{F}^{\Theta}$ represents the coefficients of the neighbouring cells (with $F$ spanning the near-neighbouring cells of cell $P$ ) and $S_{\Theta_{i j}}$ is the source term.

The numerical procedure used in the standard method to solve for the extra-stress - Eqs. (3) and (5) - was modified for the new form of stress equation based on the log-conformation tensor, Eq. (8), so that now the algorithm contains the following steps:

1. Initially, the conformation tensor $A_{i j}$, obtained from the extrastress components $\tau_{i j}$ - from Eq. (5) - is used to calculate the log-conformation tensor, $\Theta_{i j}$. For each computational cell, $A_{i j}$ is diagonalized according to Eq. (7), and all eigenvalues and eigenvectors are computed, which are used in the calculation of the orthogonal matrix, $\Omega_{i j}$, and the diagonal matrix, $D_{i j}$. The tensor $\Theta_{i j}$ is then calculated using the relation $\boldsymbol{\Theta}=\boldsymbol{\Omega}^{\mathrm{T}}(\log \mathbf{D}) \boldsymbol{\Omega}$.

2. Following the appropriate decomposition [5] of the velocity gradient, $\nabla \mathbf{u}$, and using the values of $\Omega_{i j}$, and $D_{i j}$, the traceless extensional components, $E_{i j}$, and the pure rotational component, $R_{i j}$, are calculated at each cell;

3. The evolution equation for $\Theta_{i j}$ - Eq. (10) - is solved to obtain $\Theta_{i j}$ at the new time level;

4. The conformation tensor $A_{i j}$ is recovered and the extra-stress $\tau_{i j}$ is calculated from the newly computed conformation field using Eq. (5);

5. The momentum equations are solved for each velocity component, $u_{i}$;

6. As generally the velocity components do not satisfy the continuity equation, this step of the algorithm involves a correction to $u_{i}$ and to the pressure field $p$, so that the updated velocity field $u_{i}$ and the corrected pressure field $p$ satisfy simultaneously the continuity and the momentum equations. This part of the algorithm remains unchanged and is described in detail in Oliveira et al. [21];

7. Steps 1-6 are repeated until convergence is reached (steady-state calculations), or until the desired final time is reached (unsteady calculations).

The advective term in Eq. (9) is discretised with the CUBISTA high-resolution scheme [23], formally of third-order accuracy and especially designed to deal with differential constitutive relations.

\section{Results}

In order to analyze the performance of the log-conformation formalism in comparison with the standard formulation of the extra-stress tensor, the flow past a confined circular cylinder was calculated with both formulations. For conciseness henceforth we denote by $\boldsymbol{S t r} \boldsymbol{T}$ and $\log \boldsymbol{T}$ the results obtained with the standard and $\log$-conformation methods, respectively. In all cases the $L^{1}$ norm of the residuals of the governing equations was required to be less than a tolerance of $10^{-6}$ in order to stop the time stepping procedure. In all test cases this convergence criterion was found to be sufficient to attain a converged steady-state solution. 


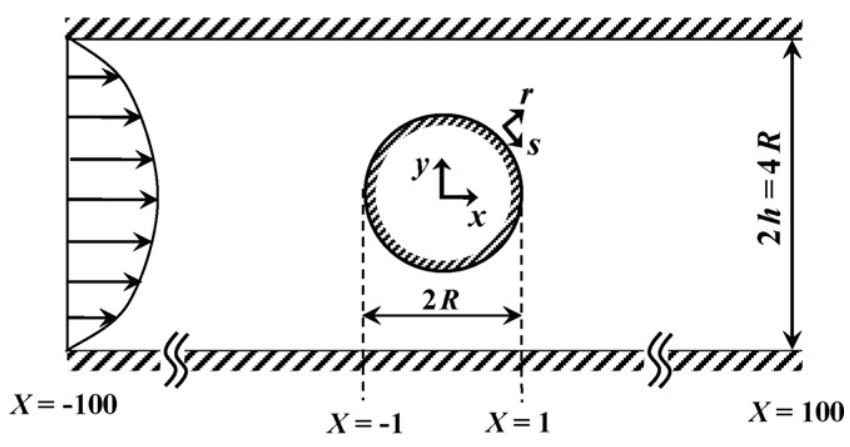

Fig. 1. Schematic representation of the flow past a confined cylinder geometry $(X=x / R)$.

\subsection{Flow geometry and computational meshes}

The geometry of the viscoelastic fluid flow past a confined cylinder in a channel is shown in Fig. 1. The ratio of channel half-height $h$ to cylinder radius $R$ is set equal to 2 , which corresponds to the benchmark $50 \%$ blockage case [27]. The computational domain is $200 R$ long, with $99 R$ upstream and $99 R$ downstream of the forward and rear stagnation points of the cylinder, respectively. The downstream length is sufficient for the flow to become fully developed and to avoid any effect of the Neumann outflow boundary condition upon the flow in the vicinity of the cylinder. Vanishing axial gradients are applied to all variables, including the pressure gradient, at the outlet plane. No-slip conditions are imposed at both the cylinder surface $(r=R: u=0, v=0)$ and the channel wall $(y= \pm h$ : $u=0, v=0$ ).

The main characteristics of the meshes $\mathrm{M} 30_{\mathrm{WR}}$ and $\mathrm{M} 60_{\mathrm{WR}}$ used in this work are given in Table 1, including the total number of cells (NC), the number of control volumes around the surface of the cylinder (NS), the number of cells placed radially from the cylinder to the channel wall (NR), and the minimum cell spacing normalized with the cylinder radius along the radial $(\Delta r)$ and the azimuthal $(\Delta s=R \Delta \theta)$ directions. All the computational meshes mapped the complete flow domain (i.e. no symmetry boundary condition was imposed along the centreline), in order to capture possible symmetry-breaking steady/unsteady phenomena $[28,29]$. The subscript WR (for wake-refined) is added to the mesh designation to denote highly refined meshes along the wake. For mesh $\mathrm{M} 30_{\mathrm{WR}}$ the minimum normalized cell spacing along the azimuthal direction is 0.0012 compared with 0.0314 for the equivalent mesh M30 used in [4]. Mesh M60 ${ }_{\mathrm{WR}}$ has twice the number of cells along both directions as mesh $\mathrm{M} 30_{\mathrm{WR}}$, with a total of 45120 cells. The number of cells along the cylinder sidewall with that mesh is 520 and the minimum normalized cell spacing along the radial and azimuthal directions is 0.004 and 0.0006 , respectively.

Results of computations are presented in two ways: as a scalar integral quantity representative of the whole flow; and as detailed profiles of stress components in the vicinity of the cylinder. The integral quantity is the dimensionless drag coefficient, $K$, calculated as

$K=\frac{1}{\eta_{0} U} \int_{S}\left(\boldsymbol{\tau}_{\text {tot }}-p \mathbf{I}\right) \cdot \mathbf{n} \cdot \mathbf{i} \mathrm{d} S$

Table 1

Main characteristics of the computational meshes.

\begin{tabular}{llllll}
\hline & NC & NR & NS & $(\Delta r / R)_{\min }$ & $(\Delta s / R)_{\min }$ \\
\hline M30 $_{\text {WR }}$ & 11280 & 30 & 260 & 0.008 & 0.0012 \\
M60 $_{\text {WR }}$ & 45120 & 60 & 520 & 0.004 & 0.0006 \\
\hline
\end{tabular}

NC: total number of cells; NR: number of cells placed radially; NS: number of cells around the cylinder surface.

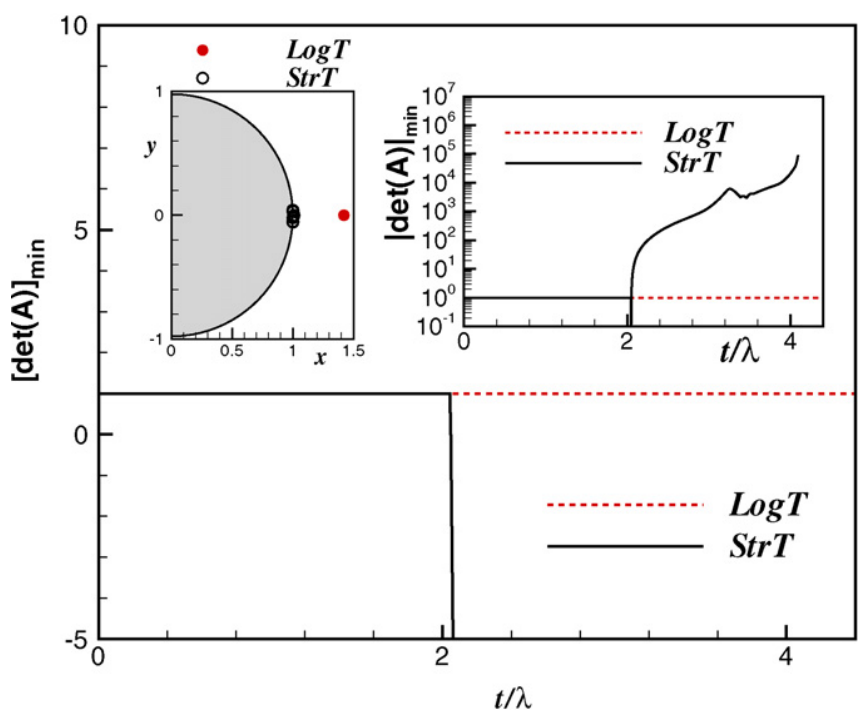

Fig. 2. Time evolution of $\operatorname{det}(\mathbf{A})_{\min }$ for creeping flow of an Oldroyd-B fluid $(\beta=0.59)$ past a cylinder. Comparison between $\operatorname{Str} T$ and $\log \boldsymbol{T}$ formulations at $D e=0.9$, calculated in mesh M60 Wr. The circles in the inset figure indicate the location where the minimum value of $\operatorname{det}(\mathbf{A})$ is observed.

where $\mathbf{I}$ is the unit tensor, $\mathbf{n}$ is the unit vector normal to the cylinder surface and $\mathbf{i}$ is the unitary vector aligned with the streamwise direction. Stress profiles are shown along the cylinder wall and on the downstream rear wake, in order to ascertain the quality of the predictions.

All the calculations were carried out at a vanishing Reynolds number, $R e=\rho U R / \eta=0$ (creeping flow conditions - imposed by dropping out the convective term in the momentum equation) and we studied the effect of the Deborah number, here defined as

$D e=\frac{\lambda U}{R}$

where $U$ and $R$ represent average velocity and the cylinder radius, respectively. This definition of Deborah number is the same used in previous works (e.g. [6-9,21,24,30]), and is consistent with taking $U$ for velocity scale and $R$ for length scale. However we note that sometimes the same dimensionless group is called Weissenberg number (e.g. [13,17]), and that a distinction between the notion of $D e$ and $W i$ is irrelevant (except perhaps for a constant multiplicative factor) when the blockage ratio $(b=R / h)$ is not varied. When such a distinction is required it is appropriate to define $W i=\lambda \dot{\gamma}_{c}$ with the characteristic shear rate based on velocity and length scales existing in the gap between the cylinder and the channel walls, thus $\dot{\gamma}_{c}=U_{\text {gap }} / h_{\text {gap }}=(3 / 2) U[h /(h-R)] /[(h-$ $R) / 2$ ], giving $W i=3 \mathrm{De} b /(1-b)^{2}$ (with $b=0.5$ as in the present study, $W i=6 \mathrm{De}$ ). The fact that our numerical results show either a steady/unsteady flow transition or difficulties to achieve mesh convergence when $D e \sim 1$, gives further support to base the level of elasticity on this definition.

The results for the Oldroyd-B model are presented in Section 4.2 and for the PTT model in Section 4.3.

\subsection{Oldroyd-B fluid}

In this section, we use the Oldroyd-B model with a solvent viscosity ratio $\beta=0.59$. Normalization of the data is done as follows: coordinates in the $x$ and $y$ directions were normalized by the cylinder radius, $R$, the velocity components by the average velocity, $U$, and the extra-stress tensor $\boldsymbol{\tau}$ and the pressure $p$ are scaled with a viscous stress, $\eta_{0} U / R$. 


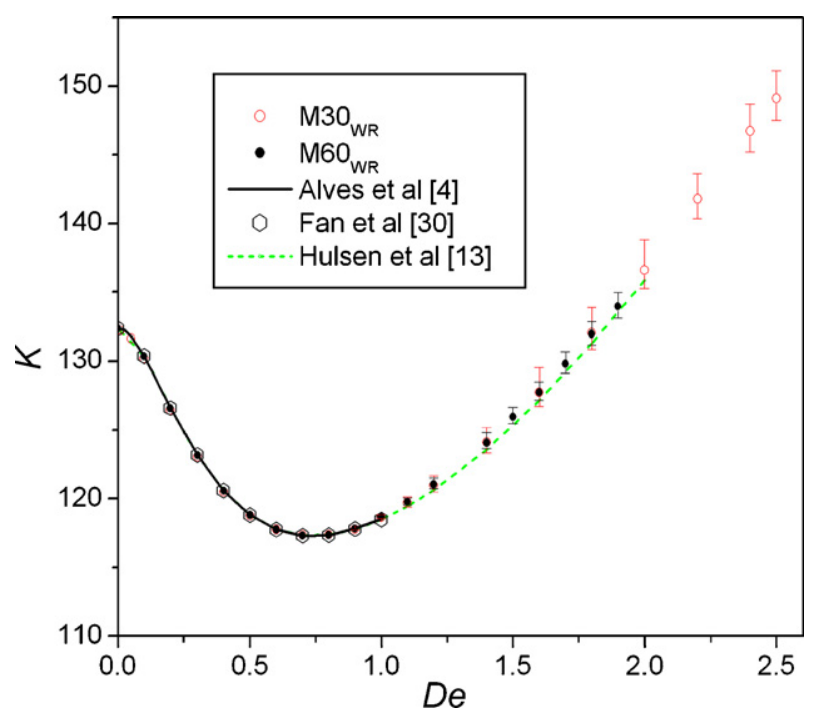

Fig. 3. Drag force coefficient for the creeping flow of an Oldroyd-B fluid ( $\beta=0.59$ ) past a cylinder. Results obtained with the $\log \boldsymbol{T}$ formulation with meshes $\mathrm{M} 3 \mathrm{~W}_{\mathrm{WR}}$ and $\mathrm{M} 60_{\mathrm{WR}}$, and comparison with data from Alves et al. [4], Hulsen et al. [13] and Fan et al. [30].

The simulations with the $\boldsymbol{S t r} \boldsymbol{T}$ formulation diverged at $D e=0.9$ with mesh $\mathrm{M} 60_{\mathrm{WR}}$. The loss of positive definiteness of $\mathbf{A}$ precedes the onset of divergence of the numerical method, as observed in Fig. 2, where the time evolution of the minimum value of the determinant of the conformation tensor along a calculation is plotted. After approximately two relaxation times, the simulation with the $\boldsymbol{S t r T}$ formulation begins to present negative values of $\operatorname{det}(\mathbf{A})_{\min }$. For the $\boldsymbol{L} \operatorname{og} \boldsymbol{T}$ formulation the value of $\operatorname{det}(\mathbf{A})_{\min }$ is, by design, always positive, thus showing no signs of violation of the positive definition criteria. We also observe that with the $\log T$ formulation $\operatorname{det}(\mathbf{A})_{\min } \geq 1$, as expected for the Oldroyd-B model [26]. It can also be observed that the location where loss of positive definiteness evolution occurs is in the rear stagnation point region, as depicted in the inset of Fig. 2. This agrees with the results of Afonso et al. [24] for uniform flow of viscoelastic fluids past a cylinder, where $\operatorname{det}(\mathbf{A})$
Table 2

Drag force coefficient for the Oldroyd-B model with $\beta=0.59$ (data in brackets indicate time-average values).

\begin{tabular}{|c|c|c|c|c|}
\hline \multirow[t]{2}{*}{ De } & \multicolumn{2}{|l|}{ StrT } & \multicolumn{2}{|l|}{$\log T$} \\
\hline & $\mathrm{M} 30_{\mathrm{WR}}$ & M60 & $\mathrm{M} 30_{\mathrm{WR}}$ & M60 ${ }_{\mathrm{WR}}$ \\
\hline 0.5 & 118.772 & 118.821 & 118.781 & 118.818 \\
\hline 0.6 & 117.768 & 117.776 & 117.778 & 117.774 \\
\hline 0.7 & 117.370 & 117.324 & 117.350 & 117.323 \\
\hline 0.8 & 117.441 & 117.370 & 117.380 & 117.364 \\
\hline 0.9 & & & 117.797 & 117.817 \\
\hline 1.0 & & & $(118.662)$ & (118.680) \\
\hline 1.1 & & & (119.740) & (119.780) \\
\hline 1.2 & & & (120.985) & (121.051) \\
\hline 1.4 & & & (124.129) & (124.092) \\
\hline 1.5 & & & (126.022) & (125.972) \\
\hline 1.6 & & & (127.759) & (127.751) \\
\hline 1.7 & & & (130.012) & (129.802) \\
\hline 1.8 & & & (132.024) & (131.937) \\
\hline 1.9 & & & (134.188) & (133.952) \\
\hline 2.0 & & & (136.580) & - \\
\hline 2.2 & & & (141.801) & - \\
\hline 2.4 & & & (146.730) & - \\
\hline 2.5 & & & (149.112) & - \\
\hline
\end{tabular}

values were minimum in the rear stagnation point at all Deborah numbers.

\subsubsection{Drag coefficient results}

The literature data for the dimensionless drag coefficient $(K)$ show some discrepancies, especially for Deborah numbers above 0.7. Alves et al. [4] used the standard method, employing highly refined non-orthogonal meshes, and the predicted drag force agrees well with values from other simulations in the literature $[13,17,18,30,31]$. In particular, their predictions of $K$ were nearly identical to those of the FEM simulations of Fan et al. [30]. The predictions of drag force of Kim et al. [31] also agreed well with the previous results up to $D e=0.9$.

The predictions of $K$ obtained in the present work are presented in Fig. 3 and in Table 2. Up to $D e=0.9$ the results agree well with the accurate data of Fan et al. [30], Alves et al. [4], Kim et al. [31] and Hulsen et al. [13]. For higher Deborah numbers, no steady-state could be attained, with the $K$ values oscillating in time with the
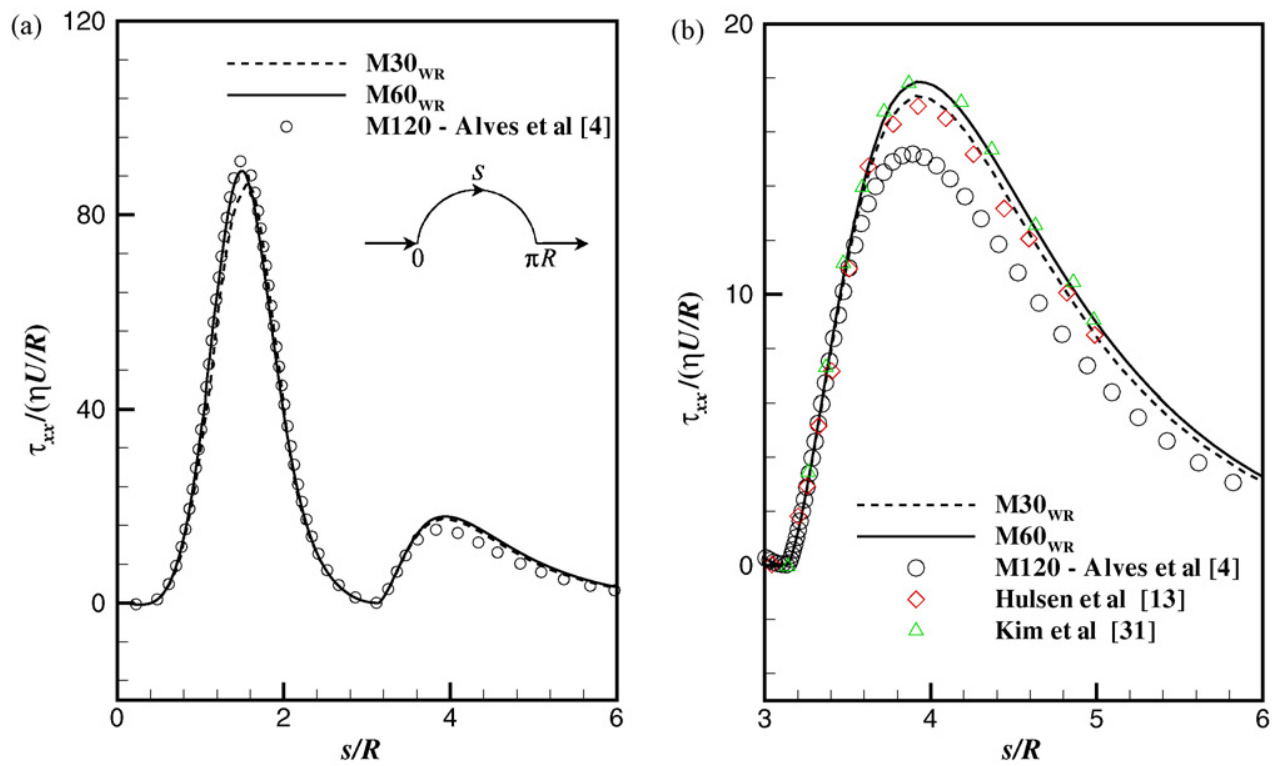

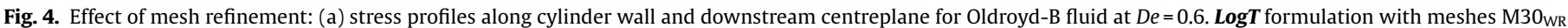
and $\mathrm{M}_{6 \mathrm{WR}}$ and comparison with data from Alves et al. [4]; (b) closer view in the rear wake of the cylinder and comparison with data from the literature. 

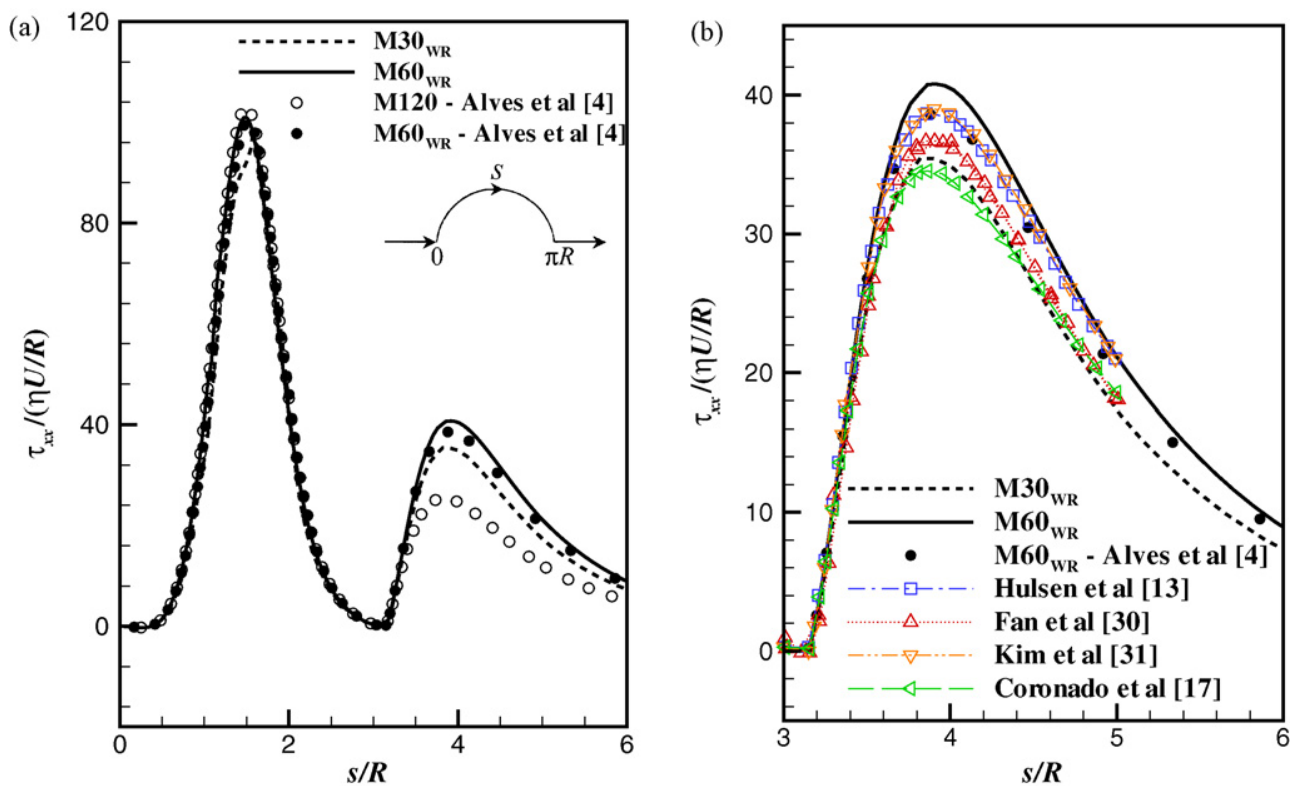

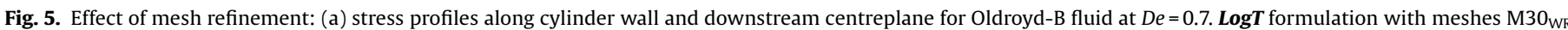
and $\mathrm{M} \mathrm{WR}_{\mathrm{WR}}$ and comparison with data from Alves et al. [4]; (b) closer view in the rear wake of the cylinder and comparison with data from the literature.

amplitude marked as error bars in Fig. 3. Here, instead of using the value of $K$ right after the onset of the transient behaviour, as used by Hulsen et al. [13], the plotted quantity is the time-average $K$ value. Although the mean values for both meshes are slightly higher than the predictions of Hulsen et al. [13], the lower bound of the oscillations are in agreement with their predictions.

\subsubsection{Stress convergence}

In terms of stress convergence with mesh refinement, it is well known that for De $>0.7$ significant discrepancies are found among the results from the literature $[4,13,17,18,30,31]$, especially in the maximum peak of normal stresses at the rear wake. Even the predictions of the extra-stresses obtained with highly refined meshes at the rear stagnation region and using high order methods are not conclusive in this respect.

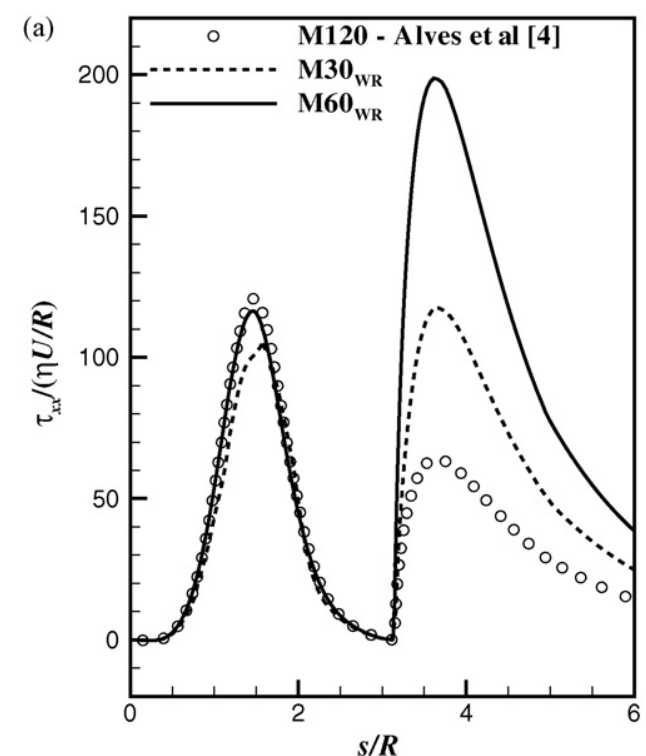

Fig. 4 shows normal stress profiles along the cylinder surface and the rear wake as obtained with different meshes at $D e=0.6$; the results are essentially mesh independent, but in the rear wake they are slightly above those of Alves et al. [4] using the StrT formulation (in this section, we use the results of Alves et al. [4] in their refined mesh (M120) to represent the StrT formulation). This difference is due to the refinement of the mesh in the wake allowing the stresses to be better resolved in meshes $\mathrm{M} 30_{\mathrm{WR}}$ and $\mathrm{M} 60_{\mathrm{WR}}$ than in mesh M120, thus showing the advantage of wake refined meshes. At $D e=0.7$ the corresponding normal stress profiles obtained with our wake refined meshes are plotted in Fig. 5, and are in good agreement with those of Alves et al. [4] obtained with the StrT formulation. In the rear wake zone there is now a more noticeable mesh dependency, with the present results obtained with the $\log \boldsymbol{T}$ formulation exhibiting higher stress peaks. The results of Alves et al. [4], Kim et

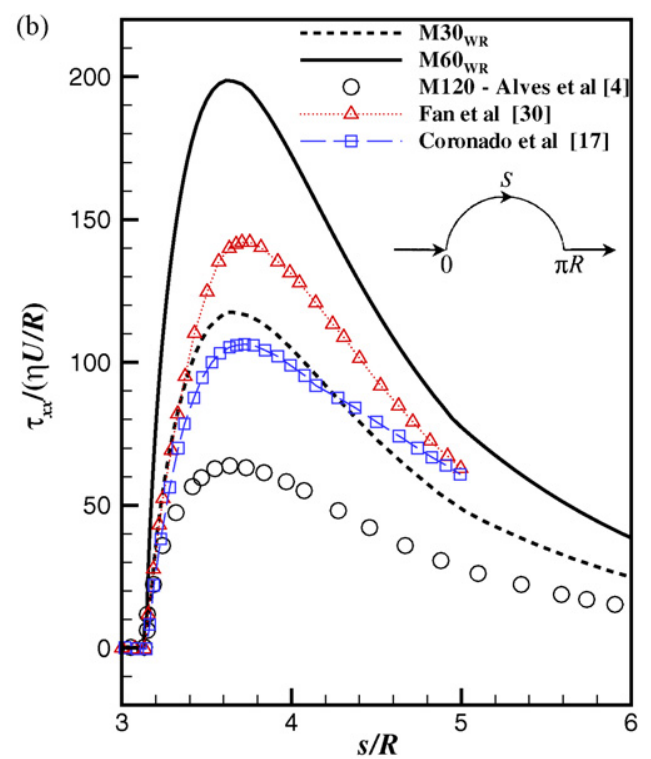

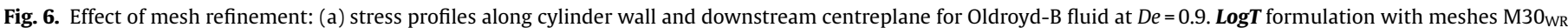
and $\mathrm{M} 60_{\mathrm{WR}}$ and comparison with data from Alves et al. [4]; (b) closer view in the rear wake of the cylinder. 


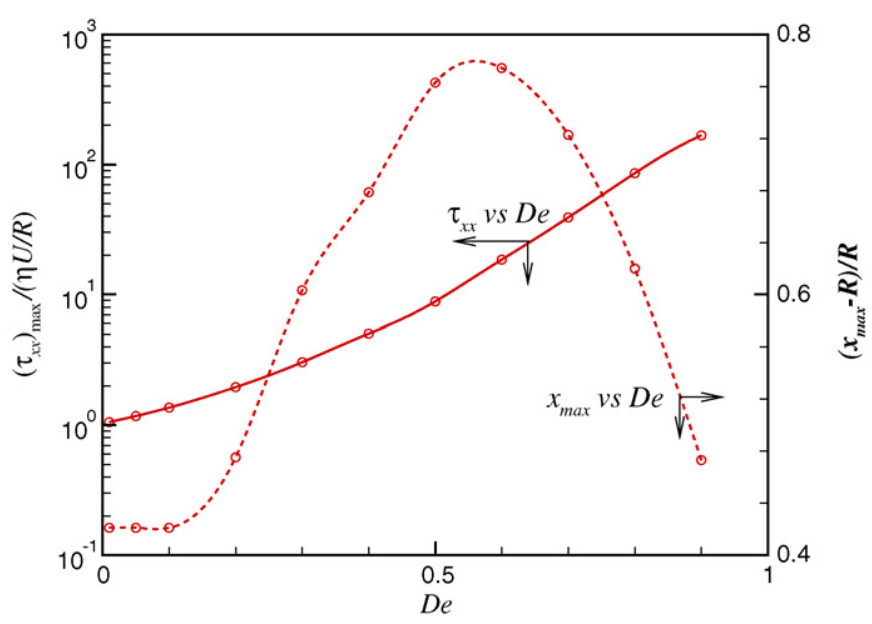

Fig. 7. Maximum value of $\tau_{x x}$ along the downstream centreline and location of peak stress, $x_{\max }$, as function of $D e$. Results obtained with the Oldroyd-B fluid in mesh M60 $\mathrm{WR}$.

al. [31] and Hulsen et al. [13] are all in close agreement, except for the maximum peak values in the wake that are slightly below those calculated here on the finest mesh.

Further increase in Deborah number to $D e=0.9$ enhances the discrepancies in the rear wake zone, as shown in Fig. 6. Although on the cylinder surface the normal stress peaks obtained with the LogT and mesh $\mathrm{M} 60_{\mathrm{WR}}$ are in close agreement with the StrT results of Alves et al. [4] for mesh M120, in the rear wake the differences are large. There is also a noticeable discrepancy in relation to the data of Fan et al. [30] and Coronado et al. [17].

Another useful observation concerns the relation between the maximum normal stress, $\left(\tau_{x x}\right)_{\max }$, along the centreline of the rear wake region and the location of peak stress, $x_{\max }$. These data are plotted in Fig. 7, and the value of $\left(\tau_{x x}\right)_{\max }$ increases nearly exponentially with $D e$ and the location $x_{\max } / R$ shows a non-monotonic behaviour, moving downstream from the rear stagnation point
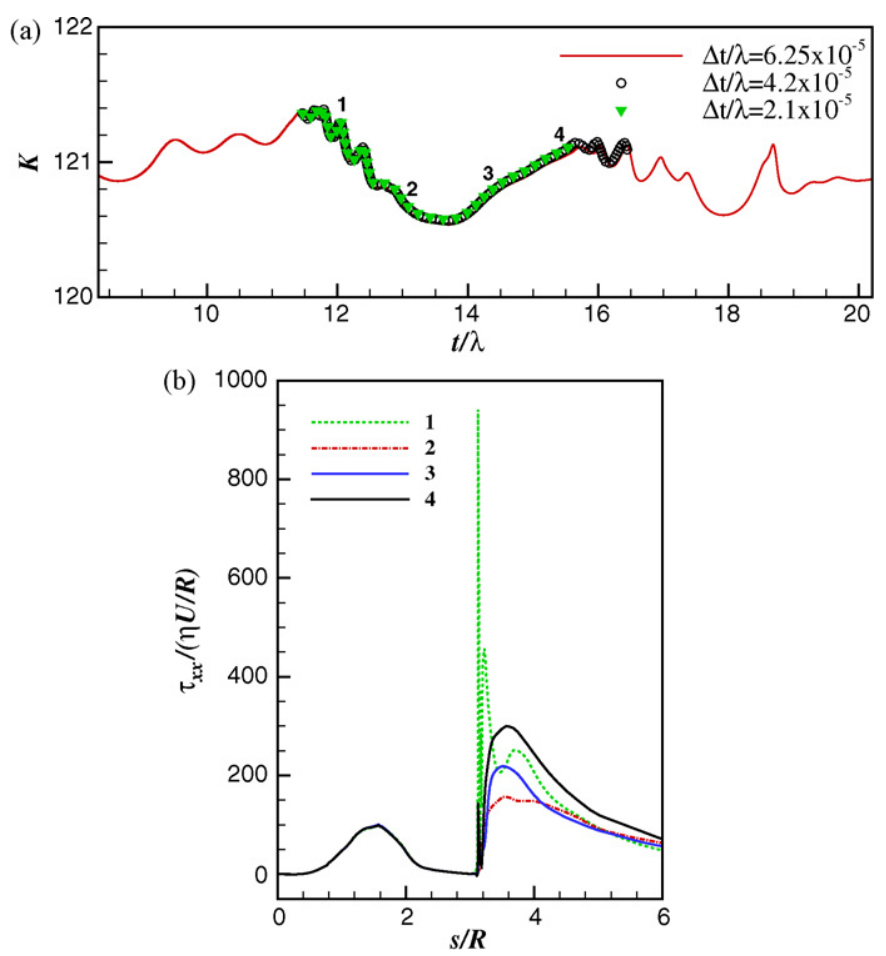

Fig. 9. Creeping flow of an Oldroyd-B fluid $(\beta=0.59$ ) past a cylinder at $D e=1.2$ (Mesh $\mathrm{M} 30_{\mathrm{WR}}$ ).(a)Time evolution of the drag force coefficient for different time steps, $\Delta t / \lambda$. (b) Axial normal stress profiles along cylinder wall and downstream centreline at the four instants marked in (a).

(from $(x-R) / R \approx 0.42$ to $(x-R) / R \approx 0.78)$ for $D e<0.6$, and shifting back towards the stagnation point at higher Deborah numbers. This upstream shift in $x_{\max } / R$ at large Deborah numbers certainly contributes to numerical difficulties, as the size of the region of peak normal stress may eventually become smaller than the local streamwise mesh resolution. Also, as $x_{\max } / R$ approaches the rear
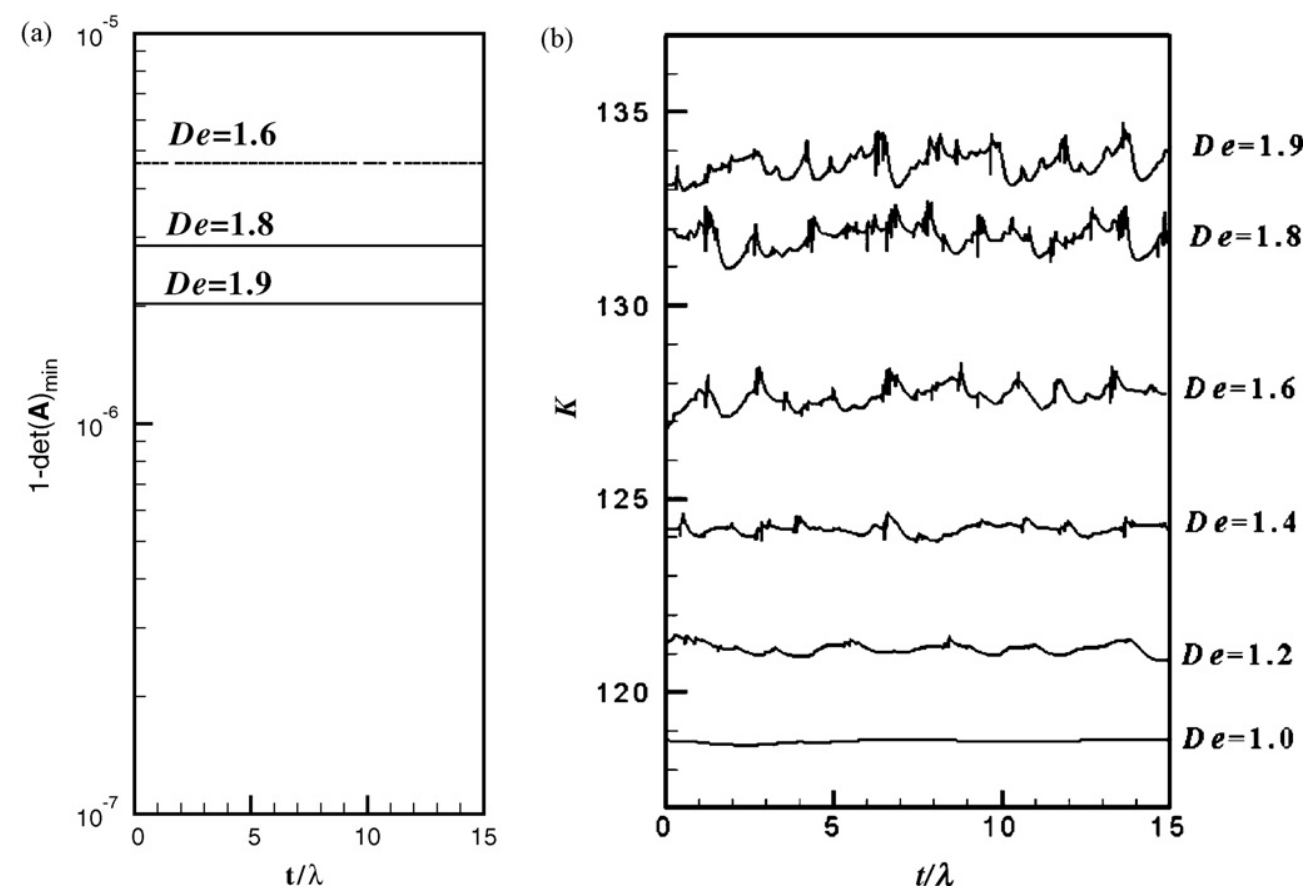

Fig. 8. Time evolution of (a) $1-\operatorname{det}(\mathbf{A})_{\min }$ and (b) drag force coefficient, calculated in mesh $M 60_{\mathrm{WR}}$. 

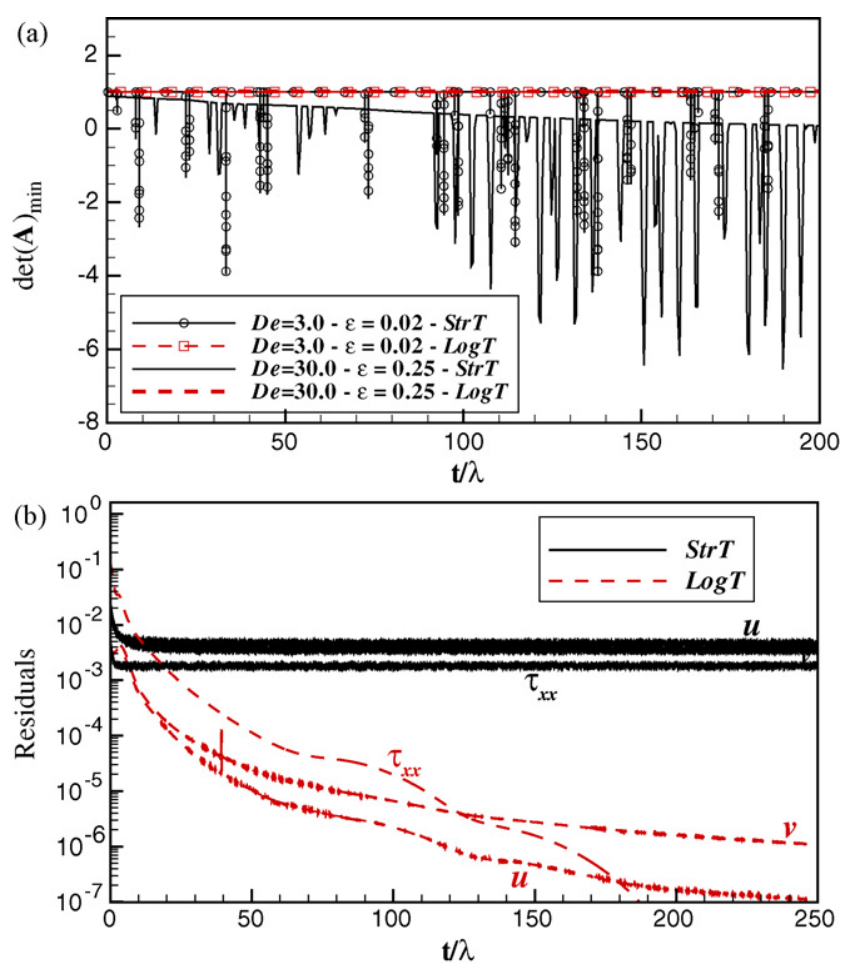

Fig. 10. Time evolution of $[\operatorname{det}(\mathbf{A})]_{\min }$ and residuals of the governing equations for the creeping flow of a PTT fluid $(\beta=0.59)$ past a cylinder calculated in mesh M30 ${ }_{\mathrm{WR}}$. (a) Comparison of $\operatorname{det}(\mathbf{A})_{\min }$ between $\boldsymbol{S t r T}$ and $\boldsymbol{L o g} \boldsymbol{T}$ formulations at $D e=3(\varepsilon=0.02)$ and $D e=30(\varepsilon=0.25)$; (b) Comparison of iterative convergence between StrT and $\log T$ formulations at $D e=30(\varepsilon=0.25)$.

stagnation point, higher residence times are observed in regions with higher strain rates, thus leading to a strong increase in the $\left(\tau_{x x}\right)_{\max }$ values as the fluid stresses are allowed to build up.

\subsubsection{High Deborah number results}

The question of whether a steady solution for cylinder flow can be attained at high De by improving numerical method accuracy and stability, is still open to debate. Fan et al. [30] and Owens et

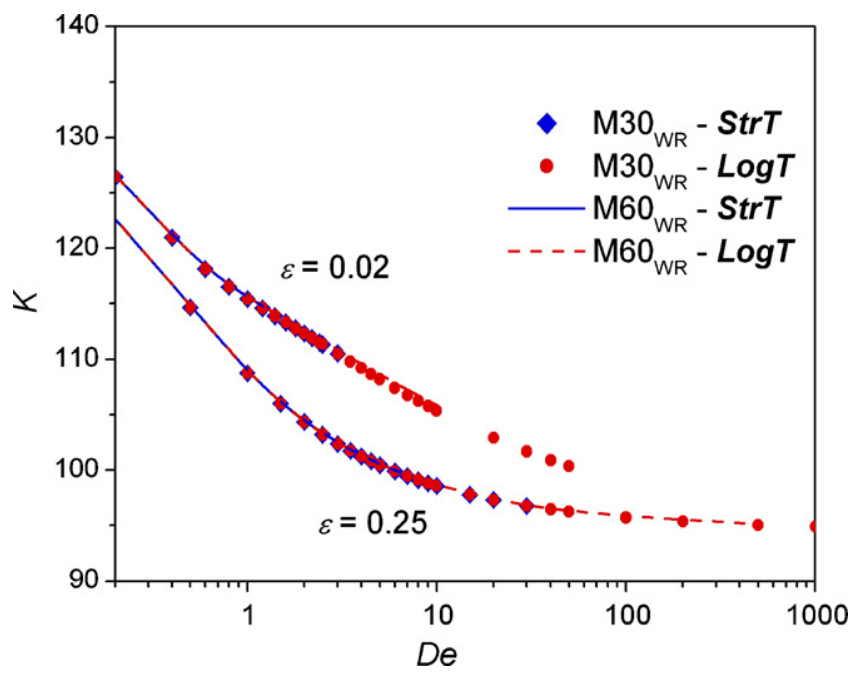

Fig. 11. Drag force coefficient for the inertialess flow of a PTT fluid $(\beta=0.59)$ past a cylinder. Results obtained with the $\boldsymbol{L o g} \boldsymbol{T}$ and $\boldsymbol{S t r} \boldsymbol{T}$ formulations with meshes $\mathrm{M} 3 \mathrm{WWR}_{\mathrm{WR}}$ and $\mathrm{M} 60_{\mathrm{WR}}$.

al. [32] argued that steady numerical solutions for the Oldroyd-B fluid flow at Deborah numbers above 0.8 are probably numerical artifacts. Recently, Bajaj et al. [33] studied the cylinder flow problem with ultra-dilute and dilute polymer solutions, modelled by the Oldroyd-B and FENE-P models, and estimated that the number of finite elements required to achieve convergence is unfeasible to obtain solutions for the Oldroyd-B model above $D e=1$, due to the steep increase in the normal stresses in the thin boundary layer in the vicinity of the cylinder. On the other hand, experimental work in this geometry with a constant-viscosity PIB polymer solution showed a first transition from steady 2D to a steady 3D flow at $D e \approx 1.3$, followed by a transition to a periodic time dependent flow at $D e \approx 1.85$ [6]. Shiang et al. [8,9] also identified experimentally a cellular structure in the cross-flow plane over a confined cylinder at $D e_{\text {crit }} \approx 0.21$, with a periodic vortical structure obtained for $D e_{\text {crit }} \approx 0.66$. The fluid used in these experiments has no noticeable shear-thinning for the range of tested shear-rates. Inspired by the experiments of McKinley et al. [6], Oliveira and Miranda [25]

Table 3

Drag force coefficient for the PTT model with $\beta=0.59$ (data in brackets indicate that full convergence was not achieved).

\begin{tabular}{|c|c|c|c|c|c|c|c|c|c|}
\hline \multirow[t]{3}{*}{ De } & \multicolumn{4}{|l|}{$\varepsilon=0.02$} & \multirow[t]{3}{*}{$D e$} & \multicolumn{4}{|l|}{$\varepsilon=0.25$} \\
\hline & \multicolumn{2}{|l|}{ StrT } & \multicolumn{2}{|l|}{$\log T$} & & \multicolumn{2}{|l|}{ StrT } & \multicolumn{2}{|l|}{$\log T$} \\
\hline & $\mathrm{M} 30_{\mathrm{WR}}$ & $\mathrm{M}^{6} 0_{\mathrm{WR}}$ & $\mathrm{M} 30_{\mathrm{WR}}$ & M60 & & $\mathrm{M} 30_{\mathrm{WR}}$ & M60 & $\mathrm{M} 30_{\mathrm{WR}}$ & M60 \\
\hline 0 & 132.231 & 132.345 & & & 0 & 132.231 & 132.345 & & \\
\hline 0.1 & 130.095 & 130.256 & 130.094 & 130.256 & 0.1 & 128.370 & 128.567 & 128.369 & 128.566 \\
\hline 0.2 & 126.428 & 126.613 & 126.427 & 126.613 & 0.5 & 114.640 & 114.805 & 114.635 & 114.805 \\
\hline 0.4 & 120.961 & 121.160 & 120.959 & 121.158 & 1.0 & 108.789 & 108.929 & 108.784 & 108.928 \\
\hline 0.6 & 118.112 & 118.312 & 118.112 & 118.310 & 1.5 & 106.022 & 106.151 & 106.024 & 106.150 \\
\hline 0.8 & 116.498 & 116.699 & 116.500 & 116.698 & 2.0 & 104.359 & 104.481 & 104.362 & 104.481 \\
\hline 1.0 & 115.405 & 115.611 & 115.409 & 115.612 & 2.5 & 103.227 & 103.344 & 103.232 & 103.345 \\
\hline 1.2 & 114.566 & 114.781 & 114.572 & 114.785 & 3.0 & 102.396 & 102.510 & 102.402 & 102.511 \\
\hline 1.4 & 113.878 & & 113.883 & 114.107 & 4.0 & 101.243 & 101.351 & 101.247 & 101.352 \\
\hline 1.6 & 113.290 & & 113.289 & 113.529 & 5.0 & 100.465 & 100.570 & 100.469 & 100.572 \\
\hline 1.8 & 112.772 & & 112.769 & 113.023 & 6.0 & 99.898 & 100.001 & 99.901 & 100.002 \\
\hline 2.0 & 112.306 & & 112.302 & 112.571 & 7.0 & 99.462 & 99.564 & 99.464 & 99.565 \\
\hline 2.5 & 111.322 & & 111.320 & 111.614 & 8.0 & 99.114 & & 99.115 & 99.216 \\
\hline 3.0 & 110.523 & & 110.505 & 110.832 & 9.0 & 98.828 & & 98.829 & 98.929 \\
\hline 4.0 & & & 109.222 & 109.605 & 10 & 98.588 & & 98.588 & 98.688 \\
\hline 5.0 & & & 108.243 & 108.662 & 20 & 97.324 & & 97.319 & 97.420 \\
\hline 6.0 & & & 107.425 & 107.905 & 50 & $(96.261)$ & & 96.244 & 96.351 \\
\hline 8.0 & & & 106.273 & 106.746 & 100 & & & 95.724 & 95.831 \\
\hline 10 & & & 105.386 & 105.888 & 500 & & & 95.057 & 95.152 \\
\hline 20 & & & 102.902 & & 1000 & & & 94.905 & \\
\hline
\end{tabular}



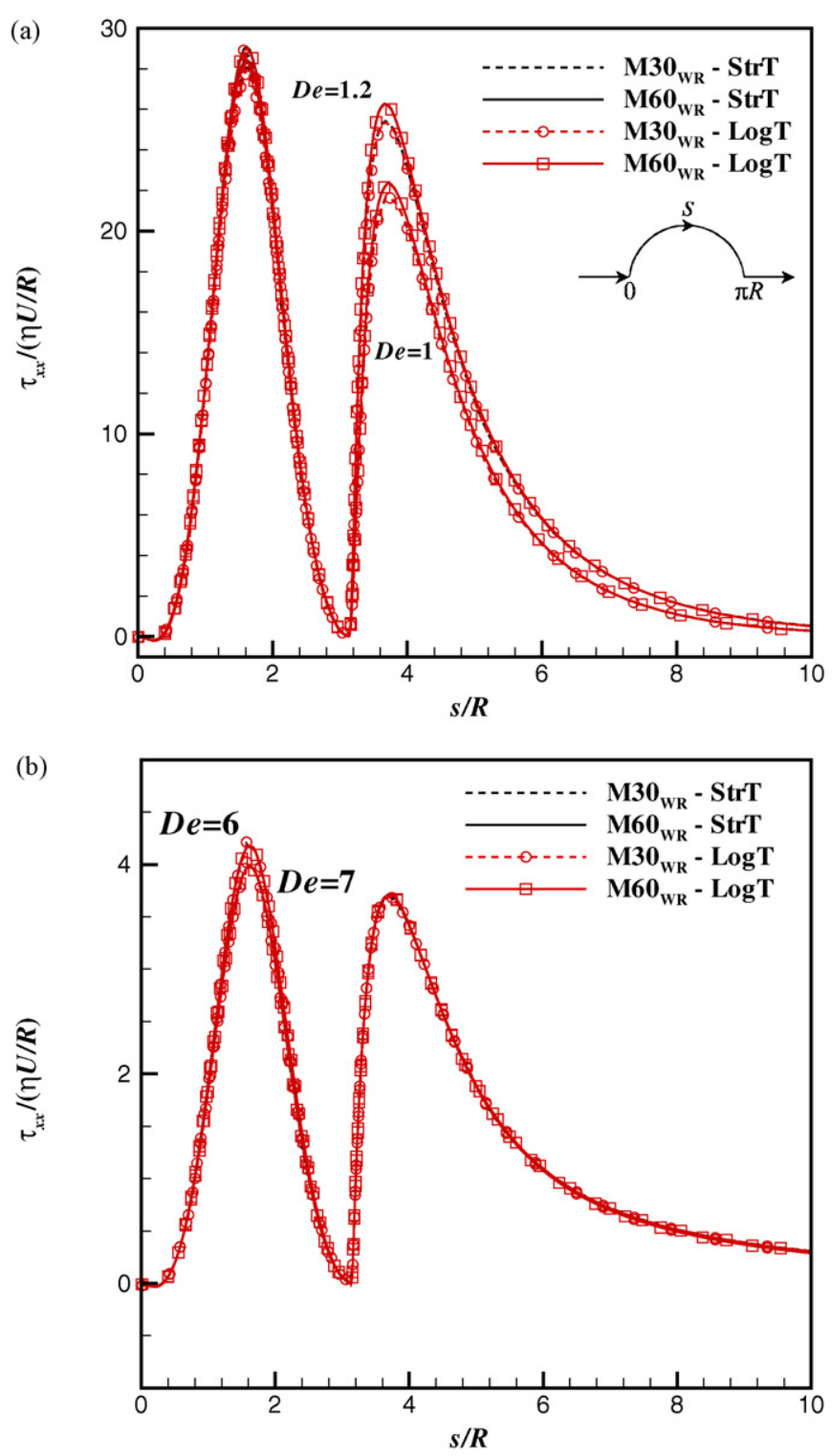

Fig. 12. Effect of mesh refinement: stress profiles along cylinder wall and downstream centreline for PTT fluid with meshes M30 $0_{\mathrm{WR}}$ and M60 ${ }_{\mathrm{WR}}$ at (a) De=1 and $D e=1.2$ with $\varepsilon=0.02$; (b) $D e=6$ and $D e=7$ with $\varepsilon=0.25$.

performed numerical simulations using the FENE-CR model and conjectured that the predicted pulsating small vortex attached to the rear stagnation point, could be the triggering mechanism leading to the formation of 3D cellular structures in the wake of the cylinder. If this is the case, then numerical results computed at high De assuming 2D flow are not representative of the real flows.

In this work, unsteady solutions were obtained for Deborah numbers from 1.0 up to 3.0 and 1.9 , for meshes $\mathrm{M} 30_{\mathrm{WR}}$ and $\mathrm{M} 60_{\mathrm{WR}}$, respectively. This represents an increase of about $90 \%$ in the maximum attainable De as one changes from the StrT to the $\log \boldsymbol{T}$ formulation. Using the $\log \boldsymbol{T}$ approach the stability requirements were always attained, as per design. Fig. 8(a) illustrates that the minimum value of $\operatorname{det}(\mathbf{A})$ is always positive, and above one [26]. As described in Section 4.2.1, the unsteady flow was also reflected in the $K$ results, and Fig. 8(b) shows the time oscillations for several $D e$, as predicted with mesh $\mathrm{M} 60_{\mathrm{WR}}$. The range of these oscillations increase with De and decrease with mesh refinement. Mesh independent results will be pursued in future work but those cal-
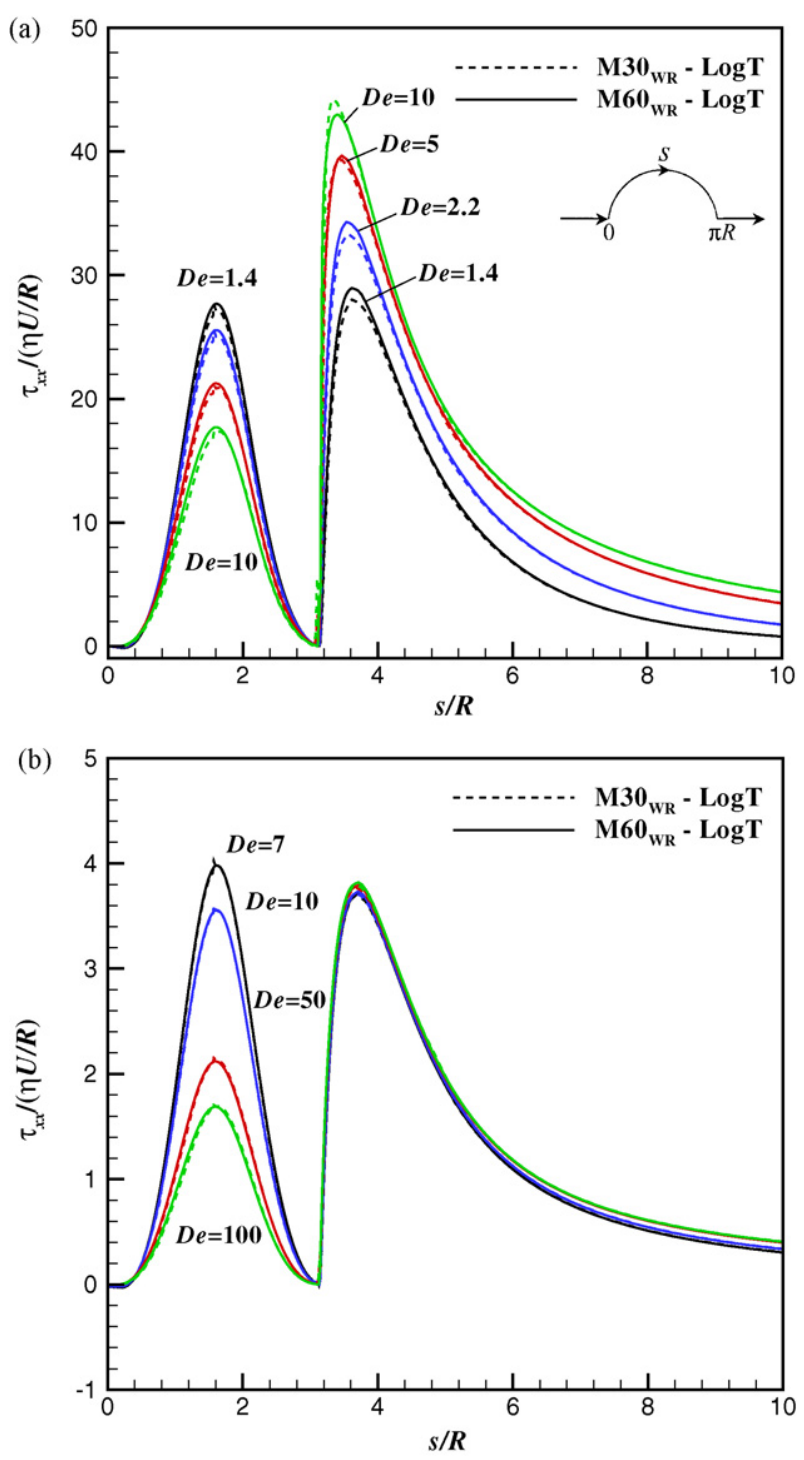

Fig. 13. Stress profiles along cylinder wall and downstream centreline for PTT fluid with meshes $\mathrm{M} 30_{\mathrm{WR}}$ and $\mathrm{M} 60_{\mathrm{WR}}$ at (a) $D e=1.4,2.2,5$ and 10 with $\varepsilon=0.02$; (b) $D e=7$, 10,50 and 100 with $\varepsilon=0.25$.

culations are very time consuming. Nevertheless, even for $D e=1.9$ the relative amplitude of the oscillations is only of about $1.4 \%$ of the time-average value.

To obtain further insights into the time-dependent flow, calculations were carried out using a second-order time discretization scheme [34] in order to improve time accuracy. Fig. 9(a) shows the time evolution of $K$ using different time steps for $D e=1.2$ and mesh $\mathrm{M} 30_{\mathrm{WR}}$. The solutions obtained with the smaller time steps $\left(\Delta t / \lambda=4 \times 10^{-5}\right.$ and $\left.\Delta t / \lambda=2 \times 10^{-5}\right)$ collapse and are independent of $\Delta t$, showing good time accuracy even if no characteristic frequency could be estimated from the numerical results of $K \mathrm{vs} t$. The normal stress profiles at four instants plotted in Fig. 9(b) show sharp increases in $\tau_{x x}$ near the rear stagnation point at some moments, thus breaking the smoothness of the stress profile in this viscoelastic flow. This reveals the major importance of the minimum mesh size in the axial direction in the stagnation point region. Although stable results were obtained up to $D e=1.8$ by Hulsen et al. [13] they also reported symptoms of stress convergence problems for $D e>0.7$, suggesting that a possible reason was that the length scale associated with the changes of the velocity gradient near the rear 
stagnation point was much smaller than the minimum mesh size in their most refined mesh. Hulsen et al. [13] also questioned the existence of a smooth solution near the cylinder and recently Renardy [35] argued that at high Deborah number flows the smoothness of viscoelastic stresses could be expected to deteriorate.

\subsection{PTT fluid}

The evolution equation for the log-conformation tensor, Eq. (8), includes the PTT model $[2,3]$ with linear stress coefficient kernel, $Y(\operatorname{tr} \mathbf{A})=1+\varepsilon(\operatorname{tr} \mathbf{A}-3)$; when $\varepsilon=0$ the Oldroyd-B model is recovered $(Y(\operatorname{tr} \mathbf{A})=1)$. This function brings in a new parameter $\varepsilon$ that imposes an upper limit to the elongational viscosity $\left(\eta_{\mathrm{E}} \sim 1 / \varepsilon\right.$ for low $\left.\varepsilon\right)$, and here the PTT model is employed with two typical values of $\varepsilon=0.02$ and 0.25 , and with solvent viscosity contribution $(\beta=0.59)$. The PTT model is shear-thinning in viscosity, in contrast to the Oldroyd-B model used in the previous sections, and has been employed in several numerical works of flow past a cylinder with both uniform and Poiseuille inlet conditions [24,36,37].

\subsubsection{Stability and drag coefficient results}

For lower values of $\varepsilon(\varepsilon=0.02)$, the simulations with the StrT formulation diverged at $D e \approx 3$ and $D e \approx 1.2$ with meshes $\mathrm{M} 30_{\mathrm{WR}}$ and $\mathrm{M} 60_{\mathrm{WR}}$, respectively. Note that when $\varepsilon \rightarrow 0$ the PTT model reduces to the Oldroyd-B model and, as observed for this latter model, a probable cause for divergence is the loss of positive definiteness, as observed in Fig. 10(a), where the determinant of the conformation tensor is presented for the near critical value of $D e=3$. The simulation with the StrT formulation has at least one negative eigenvalue of $\mathbf{A}$, leading to negative values of $\operatorname{det}(\mathbf{A})_{\min }$ and this takes place near the rear stagnation point. For the $\log \boldsymbol{T}$ formulation with $\varepsilon=0.02$, the value of $\operatorname{det}(\mathbf{A})_{\min }$ is always positive, as per design, and converged solutions up to $D e \approx 20$ and $D e \approx 10$ were obtained with meshes $\mathrm{M} 30_{\mathrm{WR}}$ and $\mathrm{M} 60_{\mathrm{WR}}$, respectively. This breakthrough in the critical Deborah number for steady flow clearly shows the advantage of using the $\log \boldsymbol{T}$ formulation. This scenario is even more outstanding in the simulations for higher values of $\varepsilon(\varepsilon=0.25)$, where the $\log \boldsymbol{T}$ formulation shows no signs of convergence problems, while for the StrT formulation positive definiteness is no longer observed for $D e \approx 30$ with mesh $\mathrm{M} 30_{\mathrm{WR}}$, as shown in Fig. 10(a). The simulations with the StrT formulation with mesh M30 $0_{\mathrm{WR}}$ still converged up to $D e \approx 200$, but the value of $\operatorname{det}(\mathbf{A})_{\min }$ was always negative with its magnitude increasing with $D e$, and with the $L^{1}$-norm of the residuals of the governing equations not attaining the required tolerance while the residuals for the equivalent $\log \boldsymbol{T}$ simulation converging up to the prescribed tolerance of $10^{-6}$, as shown in Fig. 10(b) for $D e=30$ and $\varepsilon=0.25$.

The predictions of $K$ are displayed in Fig. 11 and in Table 3. Since there is no available data in the literature for a PTT fluid at $\beta=0.59$, a direct comparison with results from both formulations is presented. Up to the critical Deborah number, the results from both approaches and values of $\varepsilon$ agree very well. For higher $D e$ the predictions of $K$ exhibit a slight mesh dependency, of approximately $0.46 \%$ $(D e=10)$ and $0.11 \%(D e=100)$ for $\varepsilon=0.02$ and $\varepsilon=0.25$, respectively.

\subsubsection{Stress convergence and high Deborah number results}

Previous works with constitutive models exhibiting shearthinning or bounded extensional viscosity, such as FENE-CR, PTT or Giesekus models [21,24,25], have shown mesh refinement not to be so crucial as with UCM and Oldroyd-B models. This observation is also valid for the predictions of $\tau_{x x}$ presented here, as shown in Fig. 12. For lower values of $\varepsilon$, the normal stress profiles along the cylinder sidewalls and in the rear region at near critical conditions ( $D e=1$ and $D e=1.2$ ) are similar for all meshes and formulations, with a slightly mesh dependency of $\tau_{x x}$ profiles in the rear region at $D e=1.2$ (c.f. Fig. 12(a)). The mesh convergence dependency weakens when the value of $\varepsilon$ is increased to 0.25 , as illustrated in Fig. 12(b), with the predictions of $\tau_{x x}$ at $D e=6$ and $D e=7$ becoming indistinguishable for all meshes and formulations, even in the rear region.

As observed in Section 4.3.1, the simulations for the PTT model using the $\boldsymbol{L} \boldsymbol{\operatorname { g g } T}$ formulation exhibit a significant breakthrough in the HWNP, with stable and convergent results obtained up to significantly higher Deborah numbers. In terms of convergence with mesh refinement, we were able to obtain mesh-independent results at low values of the extensional parameter (c.f. Fig. 13(a)), these corresponding to situations where shear-thinning is weaker. The profiles of $\tau_{x x}$ exhibit a progressive increase in magnitude and width with $D e$, both at the cylinder sidewalls and in the near wake region. For all plotted profiles, corresponding to $D e=1.4,2.2,5$ and 10 , the results obtained in meshes $\mathrm{M} 30_{\mathrm{WR}}$ and $\mathrm{M} 60_{\mathrm{WR}}$ are similar.

The high De results of Fig. 13(b), obtained with $\varepsilon=0.25$, show some new features not seen with the Oldroyd-B model. At the cylinder sidewalls for high $D e$, all $\tau_{x x}$ profiles decrease with an increase of Deborah number due to shear-thinning. This behaviour was also observed by Afonso et al. [24] for a PTT fluid in an uniform flow past a cylinder. In the near wake region the $\tau_{x x}$ profiles at high $D e$, corresponding to $D e=7,10,50$ and 100 , exhibit a small decrease in magnitude and a small increase in width downstream the cylinder. At this extensional parameter, the mesh refinement effect is negligible, in spite of the very high Deborah numbers.

\section{Conclusions}

In this work a finite-volume method was adapted to use the log-conformation formulation of Fattal and Kupferman [5] and was applied to the simulation of 2D laminar viscoelastic fluid flow around a confined cylinder, using both the Oldroyd-B and the PTT models.

In spite of the usual stress convergence problems in the confined cylinder geometry using the Oldroyd-B model, calculations with the log-conformation approach enable results to be obtained at higher Deborah numbers, as compared to the standard stress formulation, with the critical $D e$ for divergence raising from $D e \approx 1.0$ to either 1.9 or 2.5 depending on the mesh. At Deborah numbers higher than $\sim 1$ the results are unsteady without a well defined frequency, and sharp profiles are observed for $\tau_{x x}$ near the rear stagnation point breaking the smoothness of the stress profile and revealing the major importance of refinement in the axial direction at the rear stagnation region of the cylinder.

With the PTT model the stabilizing benefits of the logconformation approach are more clearly established since the range of the allowable Deborah numbers raises significantly without visible physical destabilization of the flow, which remains steady and symmetric about the $x$-axis. Quantitatively, the $\log \boldsymbol{T}$ formulation for the higher value of $\varepsilon$ tested $(\varepsilon=0.25)$ showed no signs of convergence problems on any of the meshes employed, while for the StrT formulation the violation of positive definiteness of the conformation tensor occurred at $D e \approx 7$ on the finest mesh M60 $0_{\mathrm{WR}}$. On this same mesh, and for smaller value of $\varepsilon$ tested $(\varepsilon=0.02)$, which increases the elongational viscosity and approaches the Oldroyd-B model, the critical De raised from 1.2 with the StrT formulation to 10 with the $\log \boldsymbol{T}$ formulation.

\section{Acknowledgements}

Funding from Fundação para a Ciência e a Tecnologia (FCT, Portugal) and FEDER under program POCI 2010 is gratefully acknowledged (projects POCI/EQU/59256/2004, POCI/EQU/56342/2004 
and PTDC/EME-MFE/70186/2006). A. Afonso also thanks FCT for financial support through scholarship SFRH/BD28828/2006.

\section{References}

[1] R.B. Bird, R.C. Armstrong, O. Hassager, Dynamics of Polymeric Liquids, vol. 1. Fluid Mechanics, 2nd ed., Wiley, New York, 1987.

[2] N. Phan-Thien, R.I. Tanner, A new constitutive equation derived from network theory, J. Non-Newt. Fluid Mech. 2 (1977) 353-365.

[3] N. Phan-Thien, A non-linear network viscoelastic model, J. Rheol. 22 (1978) 259-283.

[4] M.A. Alves, F.T. Pinho, P.J. Oliveira, The flow of viscoelastic fluids past a cylinder: finite-volume high-resolution methods, J. Non-Newt. Fluid Mech. 97 (2001) 207-232.

[5] R. Fattal, R. Kupferman, Constitutive laws of the matrix-logarithm of the conformation tensor, J. Non-Newt. Fluid Mech. 123 (2004) 281-285.

[6] G.H. McKinley, R.C. Armstrong, R.A. Brown, The wake instability in viscoelastic flow past confined circular cylinders, Philos. Trans. R. Soc. London A 344 (1993) 265-304.

[7] F.P.T. Baaijens, H.P.W. Baaijens, G.W.M. Peters, H.E.H. Meijer, An experimental and numerical investigation of a viscoelastic flow around a cylinder, J. Rheol. 38 (1994) 351-376.

[8] A.H. Shiang, J.C. Lin, A. Öztekin, D. Rockwell, Viscoelastic flow around a confined circular cylinder: measurements using high image-density particle image velocimetry, J. Non-Newt. Fluid Mech. 73 (1997) 29-49.

[9] A.H. Shiang, A. Öztekin, J.C. Lin, D. Rockwell, Hydroelastic instabilities in viscoelastic flow past a cylinder confined in a channel, Exp. Fluids 28 (2000) 128-142.

[10] R.G. Owens, T.N. Phillips, Computational Rheology, Imperial College Press, London, 2002.

[11] R. Fattal, R. Kupferman, Time-dependent simulation of viscoelastic flows at high Weissenberg number using the log-conformation representation, J. Non-Newt Fluid Mech. 126 (2005) 23-37.

[12] T.W. Pan, J. Hao, Numerical simulation of a lid-driven cavity viscoelastic flow at high Weissenberg numbers, C. R. Acad. Sci. Paris, Ser. I 344 (2007) 283-286.

[13] M.A. Hulsen, R. Fattal, R. Kupferman, Flow of viscoelastic fluids past a cylinder at high Weissenberg number: stabilized simulations using matrix logarithms, J. Non-Newt. Fluid Mech. 127 (2005) 27-39.

[14] Y. Kwon, Finite element analysis of planar $4: 1$ contraction flow with the tensorlogarithmic formulation of differential constitutive equations, Korea-Australia Rheol. J. 16 (2004) 183-191.

[15] S. Yoon, Y. Kwon, Finite element analysis of viscoelastic flows in a domain with geometric singularities, Korea-Australia Rheol. J. 17 (2005) 99-110.

[16] Y. Kwon, Numerical analysis of viscoelastic flows in a channel obstructed by an asymmetric array of obstacles, Korea-Australia Rheol. J. 18 (2006) 161-167.

[17] O.M. Coronado, D. Arora, M. Behr, M. Pasquali, A simple method for simulating general viscoelastic fluid flows with an alternate log-conformation formulation, J. Non-Newt. Fluid Mech. 147 (2007) 189-199.
[18] R. Guénette, A. Fortin, A. Kane, J.-F. Hétu, An adaptive remeshing strategy for viscoelastic fluid flow simulations, J. Non-Newt. Fluid Mech. 153 (2008) 34-45.

[19] J.M. Miranda, J.B.L.M. Campos, An improved numerical scheme to study mass transfer over a separation membrane, J. Membr. Sci. 188 (2001) 49-59.

[20] F. Ilinca, J.-F. Hétu, D. Pelletier, A unified finite element algorithm for twoequation models of turbulence, Computers Fluids 27 (1998) 291-310.

[21] P.J. Oliveira, F.T. Pinho, G.A. Pinto, Numerical simulation of non-linear elastic flows with a general collocated finite-volume method, J. Non-Newt. Fluid Mech. 79 (1998) 1-43.

[22] M.A. Alves, F.T. Pinho, P.J. Oliveira, Effect of a high-resolution differencing scheme on finite-volume predictions of viscoelastic flows, J. Non-Newt. Fluid Mech. 93 (2000) 287-314.

[23] M.A. Alves, P.J. Oliveira, F.T. Pinho, A convergent and universally bounded interpolation scheme for the treatment of advection, Int. J. Num. Meth. Fluids 41 (2003) 47-75.

[24] A. Afonso, M.A. Alves, P.J. Oliveira, F.T. Pinho, Uniform flow of viscoelastic fluids past a confined falling cylinder, Rheol. Acta 47 (2007) 324-348.

[25] P.J. Oliveira, A.I.P. Miranda, A numerical study of steady and unsteady viscoelastic flow past bounded cylinders, J. Non-Newt. Fluid Mech. 127 (2005) 51-66.

[26] M.A. Hulsen, Some properties and analytical expressions for plane flow of Leonov and Giesekus models, J. Non-Newt. Fluid Mech. 30 (1988) 85-92.

[27] R.A. Brown, G.H. McKinley, Report on the VIIIth international workshop on numerical methods in viscoelastic flows, J. Non-Newt. Fluid Mech. 52 (1994) 407-413.

[28] R.J. Poole, M.A. Alves, P.J. Oliveira, Purely elastic flow asymmetries, Phys. Rev. Lett. 99 (19) (2007) 164503

[29] G.N. Rocha, R.J. Poole, M.A. Alves, P.J. Oliveira, On extensibility effects in the cross-slot flow bifurcation, J. Non-Newt. Fluid Mech. 156 (2009) 58-69.

[30] Y. Fan, R.I. Tanner, N. Phan-Thien, Galerkin/least-square finite-element methods for steady viscoelastic flows, J. Non-Newt. Fluid Mech. 84 (1999) 233-256.

[31] J.M. Kim, C. Kim, K.H. Ahn, S.J. Lee, An efficient iterative solver and high precision solutions of the Oldroyd-B fluid flow past a confined cylinder, J. Non-Newt. Fluid Mech. 123 (2004) 161-173.

[32] R.G. Owens, C. Chauviére, T.N. Philips, A locally-upwinded spectral technique (LUST) for viscoelastic flows, J. Non-Newt. Fluid Mech. 108 (2002) 49-71.

[33] M. Bajaj, M. Pasquali, J.R. Prakash, Coil-stretch transition and the breakdown of computations for viscoelastic fluid flow around a confined cylinder, J. Rheol. 52 (2008) 197-223.

[34] P.J. Oliveira, Method for time-dependent simulations of viscoelastic flows: vortex shedding behind cylinder, J. Non-Newt. Fluid Mech. 101 (2001) 113-137.

[35] M. Renardy, A comment on smoothness of viscoelastic stresses, J. Non-Newt. Fluid Mech. 138 (2006) 204-205.

[36] N. Phan-Thien, H.S. Dou, Viscoelastic flow past a cylinder: drag coefficient, Comput. Meth. Appl. Mech. Eng. 180 (1999) 243-266.

[37] H.S. Dou, N. Phan-Thien, Negative wake in the uniform flow past a cylinder, Rheol. Acta 42 (2003) 383-409. 